\title{
AN ABSTRACT EXISTENCE THEOREM AT RESONANCE ${ }^{1}$
}

\author{
L. CESARI AND R. KANNAN
}

\begin{abstract}
By Schauder's fixed point theorem and alternative method (bifurcation theory) an abstract existence theorem at resonance for operational equations is proved which contains as particular cases rather different existence theorems for ordinary and partial differential equations as those of Lazer and Leach and of Landesman and Lazer.
\end{abstract}

Landesman and Lazer [8] proved, by the use of the alternative method, an existence theorem for selfadjoint elliptic Dirichlet nonlinear problems. Their theorem has since been extended, by using the alternative method or other techniques, by various authors. Detailed references may be seen in Cesari [1]-[3].

In the present paper, again in terms of the alternative method, we prove an abstract theorem, from which one can derive most of the extensions referred to above. This abstract theorem is proved here by Schauder's fixed point theorem. We restrict ourselves to the selfadjoint case here.

For extensions and variants of the abstract theorem, including nonselfadjoint cases, we refer to Cesari [4]-[6].

Let $S$ be a real separable Hilbert space with inner product $(x, y)$ and norm $\|x\|=(x, x)^{1 / 2}$. Let $E: \mathscr{D}(E) \rightarrow S$ be a linear operator with domain $\mathscr{D}(E) \subset$ $S$ and finite dimensional null space $S_{0}=\operatorname{ker} E$. Let $P: S \rightarrow S$ denote the projection operator with range $S_{0}$ and null space $S_{1}=(I-P) S$. Furthermore we assume that $S_{1}$ is also the range of $E$. Then $E$ : $\mathscr{D}(E) \cap S_{1} \rightarrow S_{1}$ is one-one and onto, and the partial inverse $H: S_{1} \rightarrow \mathscr{D}(E) \cap S$ exists as a linear operator. We assume that $H$ is a linear bounded compact operator, and that $E, P, H$ satisfy the usual axioms $\left(\mathrm{h}_{1}\right) H(I-P) E=I-P ;\left(\mathrm{h}_{2}\right) E P=$ $P E ;\left(\mathrm{h}_{3}\right) E H(I-P)=I-P$.

We have depicted here in an abstract way a situation which is rather typical for the large class of differential operators $E$ which in [1] we denote as "the selfadjoint case", while we certainly have particularized the corresponding structure. We refer to [3], [4], [5] for much more general classes of operators and corresponding structures, in particular, the nonselfadjoint case, in the alternative method.

Let $N: S \rightarrow S$ be a continuous operator in $S$ not necessarily linear. The equation

Received by the editors April 16, 1976.

AMS (MOS) subject classifications (1970). Primary 47H15, 34B15, 34C15, 35G30, 35J40.

${ }^{1}$ This research was partially supported by AFOSR Research Project 71-2122 at the University of Michigan.

(c) American Mathematical Society 1977 


$$
E x=N x, \quad x \in S,
$$

is equivalent to the system of auxiliary and bifurcation equations

$$
\begin{gathered}
x=P x+H(I-P) N x, \\
P(E x-N x)=0 .
\end{gathered}
$$

We refer to [3] for details, and we note that here, having assumed $R(P)=S_{0}$ $=\operatorname{ker} E$, the bifurcation equation reduces to $P N x=0$. Also, for $x^{*}=P x$, the auxiliary equation has the form $x=x^{*}+H(I-P) N x$. Let $L$ denote the norm of the linear operator $H(I-P): S \rightarrow S$, or $L=\|H(I-P)\|$. We denote by $(B)$ and $\left(\mathrm{N}_{0}\right)$ the following two assumptions:

(B) there is a constant $J_{0}$ such that $\|N x\| \leqslant J_{0}$ for all $x \in S$;

$\left(\mathrm{N}_{0}\right)$ there is a constant $R_{0} \geqslant 0$ such that for all $x \in S, x^{*} \in S_{0}$, with $\left\|x^{*}\right\| \geqslant R_{0}, P x=x^{*},\left\|x-x^{*}\right\| \leqslant L J_{0}$, we have $\left(N x, x^{*}\right) \leqslant 0$ (or always $\left.\left(N x, x^{*}\right) \geqslant 0\right)$.

Condition (B) is the usual situation considered in the theorems of Lazer and Leach [9], Landesman and Lazer [8], Williams [11], and in some work of DeFigueiredo [7] and others. Condition $\left(\mathrm{N}_{0}\right)$ can be shown to be implied (cf. [3]) by the specific hypotheses of the aforementioned theorems. Variants of conditions $\left(\mathrm{N}_{0}\right)$ are implied by the hypotheses of other analogous theorems (cf. [3]). Condition $\left(\mathrm{N}_{0}\right)$ was encountered by Kannan in his previous work (see [1] for references).

In [4] Cesari has considered the condition $\left(\mathrm{N}_{\varepsilon}\right)$ analogous to $\left(\mathrm{N}_{0}\right)$ where $\left(N x, x^{*}\right) \leqslant-\varepsilon\left\|x^{*}\right\|$ is required (or $\left.\left(N x, x^{*}\right) \geqslant \varepsilon\left\|x^{*}\right\|\right)$ for some $\varepsilon>0$. This condition is equally implied by the specific hypotheses of the same aforementioned theorems. Cesari has shown in [4] that condition $\left(\mathrm{N}_{\varepsilon}\right)$ implies a stronger conclusion.

THEOREM. Under hypotheses (B) and $\left(\mathrm{N}_{0}\right)$, the equation $E x=N x$ has at least one solution $x \in S$ with $\|x\| \leqslant\left(R_{0}^{2}+L^{2} J_{0}^{2}\right)^{1 / 2}$.

Proof. Let us assume we have $\left(N x, x^{*}\right) \leqslant 0$ in $\left(\mathrm{N}_{0}\right)$. Let $w=\left(w_{1}, \ldots\right.$, $w_{m}$ ) be an orthonormal base for the finite dimensional space $S_{0}=\operatorname{ker} E$ $=P S$. Then, for $x^{*} \in S_{0}$, we have $x^{*}=\sum_{1}^{m} c_{i} w_{i}$, or briefly $x^{*}=c w$, where $c=\left(c_{1}, \ldots, c_{m}\right) \in R^{m}, c_{i}=\left(x^{*}, w_{i}\right), i=1, \ldots, m$, and $\left\|x^{*}\right\|=|c|=$ $\left(\Sigma_{1}^{m} c_{i}^{2}\right)^{1 / 2}$. Then system $(2,3)$ with $P x=x^{*}$ can be written in the form $x=c w+H(I-P) N x,(P N x, w)=0$, where in the last relation $(P N x, w)$ simply denotes the $m$-vector $\left[\left(P N x, w_{i}\right), i=1, \ldots, m\right]$. Let

$$
L=\|H(I-P)\|=\|H\|, \quad J=\omega_{0},
$$

and let $R_{1}, R_{2}, R, \varepsilon$ be constants so chosen that

$$
0 \leqslant R_{0} \leqslant R_{1}<R_{2}<R, \quad R_{2}+J_{0} \leqslant R, \quad 0<\varepsilon<R_{1} / 2 .
$$

We shall consider the transformation $T:(x, c) \rightarrow(\bar{x}, \bar{c})$ defined by

$$
\begin{gathered}
T: \bar{x}=c w+H(I-P) N x, \quad \bar{c}=c+F(\bar{x}, c), \\
(x, c) \in \mathcal{C}=\left[(x, c)\left|x \in S, c \in R^{m},\|x\| \leqslant R+J,\right| c \mid \leqslant R\right],
\end{gathered}
$$


where $x^{*}=c w=\sum_{1}^{m} c_{i} w_{i}, \bar{x}^{*}=\bar{c} w=\bar{c}_{1} w_{1}+\cdots+\bar{c}_{m} w_{m}, c, \bar{c} \in R^{m}$, and $F(\bar{x}, c)=\left(F_{1}, \ldots, F_{m}\right)$ is explicitly given below. Note that

$$
\bar{x}^{*}=x^{*}+F(\bar{x}, c) w=x^{*}+\sum_{1}^{m} F_{i} w_{i} \text {. }
$$

Here, for $0 \leqslant|c| \leqslant R_{1}$ we take $F(\bar{x}, c)=(P N \bar{x}, w)$, or $\bar{x}^{*}=x^{*}+P N \bar{x}$. For $R_{2} \leqslant|c| \leqslant R$, or $R_{2} \leqslant\left\|x^{*}\right\|=|c| \leqslant R$, we take

$$
\begin{aligned}
F(\bar{x}, c) & =\left[\left(P N \bar{x}, x^{*}\right)-\varepsilon\|P N \bar{x}\|\right]\left(2 J_{0}\left\|x^{*}\right\|\right)^{-1}\left(x^{*}, w\right), \text { or } \\
\bar{x}^{*} & =x^{*}+\left[\left(P N \bar{x}, x^{*}\right)-\varepsilon\|P N \bar{x}\|\right]\left(2 J_{0}\left\|x^{*}\right\|\right)^{-1} x^{*} .
\end{aligned}
$$

For $R_{1} \leqslant\left\|x^{*}\right\|=|c| \leqslant R_{2}$ we take

$$
F(\bar{x}, c)=\lambda(P N \bar{x}, w)+(1-\lambda)\left[\left(P N \bar{x}, x^{*}\right)-\varepsilon\|P N \bar{x}\|\right]\left(2 J_{0}\left\|x^{*}\right\|\right)^{-1}\left(x^{*}, w\right),
$$

or

$$
\begin{gathered}
\bar{x}^{*}=x^{*}+\lambda P N \bar{x}+(1-\lambda)\left[\left(P N \bar{x}, x^{*}\right)-\varepsilon\|P N \bar{x}\|\right]\left(2 J_{0}\left\|x^{*}\right\|\right)^{-1} x^{*} \\
\lambda=\left(R_{2}-R_{1}\right)^{-1}\left(R_{2}-|c|\right), \quad 0 \leqslant \lambda \leqslant 1 .
\end{gathered}
$$

We have $\bar{c}=c$, or $\bar{x}^{*}=x^{*}$, if and only if $F(\bar{x}, c)=0$. For $|c| \leqslant R_{1}$ we have $F=0$ if and only if $P N \bar{x}=0$. For $R_{2} \leqslant|c| \leqslant R$ we have

$$
F w=\left[\left(P N \bar{x}, x^{*}\right)-\varepsilon\|P N \bar{x}\|\right]\left(2 J_{0}\left\|x^{*}\right\|\right)^{-1} x^{*}
$$

with $x^{*} \neq 0$ and $\left(P N \bar{x}, x^{*}\right)-\varepsilon\|P N \bar{x}\| \leqslant-\varepsilon\|P N \bar{x}\|$, and again $F=0$ if and only if $P N \bar{x}=0$. For $R_{1}<|c|<R_{2}$, hence $0<\lambda<1$, we have

$$
\left(F w, x^{*}\right)=\lambda\left(P N \bar{x}, x^{*}\right)+(1-\lambda)\left[\left(P N \bar{x}, x^{*}\right)-\varepsilon\|P N \bar{x}\|\right]\left(2 J_{0}\left\|x^{*}\right\|\right)^{-1}\left\|x^{*}\right\|^{2}
$$

where $\left(P N \bar{x}, x^{*}\right) \leqslant 0$, (cf. below), $\left(P N \bar{x}, x^{*}\right)-\varepsilon\|P N \bar{x}\|<-\varepsilon\|P N \bar{x}\|$, and again $F=0$ if and only if $P N \bar{x}=0$. Thus, concerning the transformation $T \mid \mathcal{C}$, we have $(x, c)=T(x, c)$ if and only if $x=x^{*}+H(I-P) N x$ and $P N x=0$, that is, the fixed points $(x, c)$ of $T$ on $C$ correspond exactly to the solutions of system $(2,3)$, that is, to the solutions of the original equation $E x=N x$.

Let us prove that $T$ maps $\mathcal{C}$ into itself. First, for $(x, c) \in \mathcal{C}$ we have $x^{*}=c w,\left\|x^{*}\right\|=\|c w\|=|c| \leqslant R$, and

$$
\|\bar{x}\| \leqslant\|c w\|+\|H(I-P) N x\| \leqslant|c|+L J_{0} \leqslant R+J .
$$

Now we have $P \bar{x}=c w=x^{*}$,

$$
\|\bar{x}-P \bar{x}\|=\left\|\bar{x}-x^{*}\right\|=\|H(I-P) N x\| \leqslant L J_{0}=J .
$$

Thus, $\left(P N \bar{x}, x^{*}\right)=\left(N \bar{x}, x^{*}\right) \leqslant 0$ for $\left\|x^{*}\right\| \geqslant R_{0}$, in particular, for $R_{1} \leqslant|c|=$ $\left\|x^{*}\right\| \leqslant R$.

For $|c| \leqslant R_{1}$ we have $\bar{c}=c+F(\bar{x}, c)=c+(P N \bar{x}, w)$, hence

$$
|\bar{c}| \leqslant|c|+\|P N \bar{x}\| \leqslant R_{1}+J_{0} \leqslant R_{2}+J_{0} \leqslant R .
$$

For $R_{2} \leqslant|c| \leqslant R$ we have 


$$
\bar{x}^{*}=\left\{1+\left[\left(P N \bar{x}, x^{*}\right)-\varepsilon\|P N \bar{x}\|\right]\left(2 J_{0}\left\|x^{*}\right\|\right)^{-1}\right\} x^{*}=\Lambda x^{*},
$$

where $\Lambda$ is the number in braces, $\left(P N \bar{x}, x^{*}\right) \leqslant 0,\left|\left(P N \bar{x}, x^{*}\right)\right| \leqslant J_{0}\left\|x^{*}\right\|$, $\|P N \bar{x}\| \leqslant J_{0},\left\|x^{*}\right\| \geqslant R_{2}>R_{1}, \varepsilon / R_{1} \leqslant \frac{1}{2}, \frac{1}{4}=1-\frac{1}{2}-\frac{1}{4} \leqslant \Lambda \leqslant 1$. Thus, $\bar{x}^{*}$ is on the segment between $x^{*}$ and $x^{*} / 4$, and $|\bar{c}| \leqslant|c| \leqslant R$.

For $R_{1}<c<R_{2}$ we have $0<\lambda<1$,

$$
\bar{x}^{*}=\lambda(P N \bar{x})+\left\{1+(1-\lambda)\left[\left(P N \bar{x}, x^{*}\right)-\varepsilon\|P N \bar{x}\|\right]\left(2 J_{0}\left\|x^{*}\right\|\right)^{-1}\right\} x^{*},
$$

and $\left\|\bar{x}^{*}\right\| \leqslant\|P N \bar{x}\|+\left\|\{\cdots\} x^{*}\right\| \leqslant J_{0}+R_{2} \leqslant R$. We have proved that $T: \mathcal{C} \rightarrow$ C.

Let us prove that $T$ is compact. For this we consider any (bounded) sequence $\left(x_{k}, c_{k}\right), k=1,2, \ldots$, of points of $C$. Then the sequence $N x_{k}$ is bounded, actually,

$$
\left\|(I-P) N x_{k}\right\| \leqslant\left\|N x_{k}\right\| \leqslant J_{0}, \quad\left\|y_{k}\right\|=\left\|H(I-P) N x_{k}\right\| \leqslant L J_{0},
$$

and since $H$ is compact, there is a subsequence, say still [ $k$ ], so that $y_{k}$ is convergent in $S$. Certainly $x_{k}^{*}=c_{k} w, F\left(\bar{x}_{k}, c_{k}\right)=d_{k} w$ are bounded sequences, actually $\left|c_{k}\right| \leqslant R,\left|d_{k}\right| \leqslant R$, both $c_{k}$ and $d_{k}$ in $R^{m}$, a finite dimensional space. Thus, we can extract the subsequence, say still [k], in such a way that $c_{k}, d_{k}$ are convergent in $R^{m}$, and then $\bar{x}_{k}=x_{k}^{*}+y_{k}, \bar{c}_{k}=c_{k}+d_{k}$ are convergent in $S$ and $R^{m}$, respectively. We have proved that $T$ is compact.

By Schauder's fixed point theorem, $T: \mathcal{C} \rightarrow \mathcal{C}$ has at least one fixed point $(x, c)=T(x, c)$. If $\left(N x, x^{*}\right) \geqslant 0$, then the proof is the same provided we take $-\left(P N \bar{x}, x^{*}\right)$ instead of $\left(P N \bar{x}, x^{*}\right)$ in the definition of $F$.

\section{REFERENCES}

1. L. Cesari, The alternative method in nonlinear analysis, Internat. Conf. on Differential equations (H. Antosiewicz, editor), Academic Press, New York, 1975, pp. 95-148.

2. Nonlinear oscillations in the frame of alternative methods, Internat. Conf. on Dynamical Systems, Vol. 1 (Providence, R.I.), Academic Press, New York, 1976, pp. 29-50.

3. __ Functional analysis and nonlinear differential equations, Dynamical Systems (Cesari, Kannan and Schuur, editors), Dekker, New York, 1976.

4. __ An abstract existence theorem across a point of resonance, Internat. Sympos. on Dynamical Systems (Gainesville, Fla., March 24-26, 1976), Academic Press, New York (to appear).

5. Nonlinear oscillations across a point of resonance for nonselfadjoint systems, J. Differential Equations (to appear).

6. __ Nonlinear problems across a point of resonance for nonselfadjoint systems, Nonlinear Analysis, A volume in honor of E. H. Rothe, Academic Press, New York (to appear).

7. D. G. De Figueiredo, The Dirichlet problem for nonlinear elliptic equations: a Hilbert space approach, Partial Differential Equations and Related Topics, Lecture Notes in Math., vol. 446, Springer-Verlag, Berlin and New York, 1975, pp. 144-165.

8. E. M. Landesman and A. C. Lazer, Nonlinear perturbations of linear elliptic boundary value problems at resonance, J. Math. Mech. 19 (1969/70), 609-623. MR 42 \#2171.

9. A. C. Lazer and D. E. Leach, Bounded perturbations of forced harmonic oscillations at resonance, Ann. Mat. Pura Appl. (4) 82 (1969), 49-68. MR 40 \# 2972. 
10. S. A. Williams, A connection between the Cesari and Leray-Schauder methods, Michigan Math. J. 15 (1968), 441-448. MR 38 \#5085.

11. , A sharp sufficient condition for solution of a nonlinear elliptic boundary value problem, J. Differential Equations 8 (1970), 580-586. MR 42 \#2169.

Department of Mathematics, University of Michigan, Ann Arbor, Michigan 48104

Department of Mathematics, University of Missouri, St. Louis, Missouri 63121 